\title{
Combining Prostate-Specific Antigen Parameters With Prostate Imaging Reporting and Data System Score Version 2.0 to Improve Its Diagnostic Accuracy
}

\author{
Sasanka Kumar Baruaha, Nabajeet Das ${ }^{\mathrm{a}, \mathrm{b}}$, Saumar Jyoti Baruah ${ }^{\mathrm{a}}$, T.P. Rajeev ${ }^{\mathrm{a}}$, \\ Puskal Kumar Bagchia ${ }^{a}$, Debanga Sharma ${ }^{a}$, Mandeep Phukan ${ }^{\mathrm{a}}$
}

\begin{abstract}
Background: Any non-invasive test that can predict the absence of prostate cancer (PCa) or absence of clinically significant PCa (CSP$\mathrm{Ca}$ ) is necessary, as it can reduce the number of unnecessary biopsies in patients with gray zone prostate-specific antigen (PSA, 4 - $10 \mathrm{ng}$ / $\mathrm{mL}$ ). This study evaluated the diagnostic performance of free PSA $\%$ and PSA density (PSAD), and Prostate Imaging Reporting and Data System (PIRADS) score (version 2.0) alone and combined in predicting CSPCa in patients with PSA between 4 and $10 \mathrm{ng} / \mathrm{mL}$.

Methods: This prospective study included a total of 104 consecutive patients with lower urinary tract symptoms (LUTS) and serum PSA between 4 and $10 \mathrm{ng} / \mathrm{mL}$, with or without abnormal digital rectal examination (DRE) findings or any hypoechoic lesion on ultrasound sonography of prostate and without prior transrectal ultrasound (TRUS) biopsy of prostate. PIRADS score was calculated using multi-parametric magnetic resonance imaging (mp-MRI) before TRUS biopsy of prostate. Relationships among PIRADS score, PSAD, free PSA \% and presence of CSPCa in TRUS biopsy were statistically analyzed.
\end{abstract}

Results: In patients with CSPCa, significantly higher median age ( $\mathrm{P}$ $=0.001)$, PSA level $(\mathrm{P}<0.001)$, PSAD $(\mathrm{P}<0.001)$ and significantly lower prostate volume $(\mathrm{P}<0.001)$ and free PSA $\%$ were observed as compared to patients with non-CSPCa. Significantly higher proportion of patients with CSPCa showed PIRADS positive test compared to those with non-CSPCa $(86.4 \%$ vs. $53.3 \%, \mathrm{P}<0.001)$. Cut-off values for PSAD and free PSA $\%$ were $0.12 \mathrm{ng} / \mathrm{mL}^{2}$ and $25 \%$, respectively. Age, PSAD and free PSA \% were significant predictors of PCa, while age and PSAD were significant predictors of CSPCa. Criteria 2, 3 and 4 demonstrated higher specificity and positive predictive value (PPV) in predicting CSPCa as compared to criterion 1 . The overall accuracies of criterion $1,2,3$ and 4 were $64.42 \%, 85.58 \%, 80.77 \%$ and $79.81 \%$, respectively. The area under the curve (AUC) values of criterion 2, 3 and 4 were higher $(0.827,0.732$ and 0.792$)$ than criterion $1(0.665)$.

Manuscript submitted September 2, 2019, accepted October 21, 2019

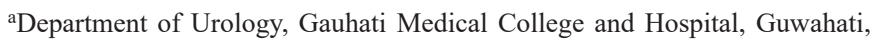
India

${ }^{\mathrm{b}}$ Corresponding Author: Nabajeet Das, Department of Urology, Gauhati Medical College and Hospital, Guwahati, India. Email: nabajeetdas14@gmail.com

doi: https://doi.org/10.14740/wjon1230
Conclusion: Using PIRADS score for predicting CSPCa as a screening test, criteria 2, 3 and 4 have much higher diagnostic performance and present accuracy of mp-MRI to predict CSPCa can be increased with addition of PSAD and free PSA $\%$.

Keywords: PIRADS score; PSA density; Free PSA\%; Clinically significant prostate cancer; mp-MRI; Prostate biopsy

\section{Introduction}

Prostate cancer (PCa) is most common malignancy found in men and according to GLOBOCAN 2018, estimated incidence of $\mathrm{PCa}$ was $7.1 \%$ and mortality rate due to $\mathrm{PCa}$ was $3.8 \%$ in the world [1]. Prostate-specific antigen (PSA) screening has been shown as a better diagnostic tool in identifying PCa and to be associated with decreased $\mathrm{PCa}$-specific mortality rates [2]. Transrectal ultrasound (TRUS)-guided biopsy of prostate is another diagnostic tool for early identification of PCa. However, both these techniques identify many indolent tumors and radical prostatectomy performed for these patients does not bring survival benefits and subsequently have adverse impact on quality of life [3].

Men having serum PSA level between 4 and $10 \mathrm{ng} / \mathrm{mL}$ are termed as "gray zone" wherein TRUS-guided prostate biopsies are not required and clinically insignificant PCa may be overtreated [4]. Further, biopsy of prostate is associated with risks of sepsis and bleeding, besides being an invasive and a costly test [5]. Therefore, any non-invasive tests that can confidently predict the absence of $\mathrm{PCa}$ or absence of clinically significant $\mathrm{PCa}(\mathrm{CSPCa})$ are necessary, as it can reduce the number of unnecessary biopsies in patients with gray zone PSA.

PSA parameters like free PSA \% and PSA density (PSAD) can facilitate diagnosis of $\mathrm{PCa}$ in patients with gray zone PSA $[5,6]$. The increased availability of magnetic resonance imaging (MRI) of prostate, its morphologic and different functional imaging modalities along with its greater standardization increased its performance in detecting, localizing and staging PCa [7, 8]. In 2012, the European Society of Urogenital Radiology (ESUR) published clinical guidelines for multi-parametric MRI (mp-MRI) along with a structured reporting system called the "Prostate Imaging Reporting and Data System (PI- 
RADS)" [9, 10]. During the later years, the ESUR developed an updated version of PIRADS version 1, known as PIRADS version 2.0 [9]. The PIRADS assesses the probability of finding CSPCa on a five-point Likert scale for each lesion $[11,12]$.

A considerable number of studies have commented on high negative predictive value (NPV) of mp-MRI for detecting CSPCa using 12-core biopsy, saturation or radical prostatectomy specimen as reference tests. The rationale behind performing this study is that, many studies have reported low specificity and low positive predictive value (PPV) of PIRADS score $[3,13-15]$. If the specificity and PPV of PIRADS score can be increased, it can help in predicting lesions having CSPCa in pre-biopsy MRI or prevent a biopsy altogether in patients having contraindication to TRUS biopsy.

Therefore, the present study aimed to examine the diagnostic significance of PSA parameters like free PSA $\%$ and PSAD, and PIRADS score version 2.0 in predicting CSPCa in patients having PSA between 4 and $10 \mathrm{ng} / \mathrm{mL}$. Additionally, this study evaluated whether combination of PSA parameters and PIRADS score version 2.0 can affect the diagnostic accuracy of mp-MRI for predicting CSPCa.

\section{Materials and Methods}

The present study was a prospective study conducted in the Department of Urology and Renal Transplantation, Gauhati Medical College and Hospital, Guwahati, India between April 2017 and October 2018. The study protocol was approved by Institutional Review Board and Hospital Ethics Committee and the study was in accordance with the Declaration of Helsinki.

Inclusion criteria were patients above age of 40 years who attended urology outpatient department (OPD) with lower urinary tract symptoms (LUTS) having clinical features suspicious of harboring prostate malignancy whose serum PSA was $\geq 4 \mathrm{ng} /$ $\mathrm{mL}$ but $\leq 10 \mathrm{ng} / \mathrm{mL}$, with or without abnormal digital rectal examination (DRE) findings or any hypoechoic lesion on prostate ultrasound sonography (USG); with no prior TRUS biopsy of prostate. Whereas, exclusion criteria were patients having prostatitis or urinary tract infection, and taking a $5 \alpha$ reductase inhibitors or those who have history of intake within 6 months, patients with more than 1 month interval between pre-biopsy mp-MRI and TRUS-guided biopsy, patients who were unfit to MRI (patients with estimated glomerular filtration rate (eGFR) $\leq 50 \mathrm{~mL} /$ $\mathrm{min} / 1.73 \mathrm{~m}^{2}$, pacemaker implant, metallic hip, or extensive pelvic orthopaedic metal work) and patients who were unfit to undergo TRUS biopsy (prostatitis, uncorrected coagulopathy).

The following variables were used in the study. Age was defined between 40 and 90 years. PSA gray zone was defined as between 4 and $10 \mathrm{ng} / \mathrm{mL}$. Prostate volume was measured using transabdominal ultrasound by using formula: $0.523 \times$ transverse diameter $\times$ antero-posterior diameter $\times$ longitudinal diameter. PSAD was defined as PSA value in $n g /$ volume in $\mathrm{mL}^{3}$. Free PSA $\%$ was defined as free PSA/total PSA $\times 100$. PIRADS score and presence of clinically significant cancer are defined in subsequent paragraphs.

A detailed medical history and physical examination were performed. DRE was done and presence of suspicious nodule or prostatitis was noted. Urine routine examination, urine culture sensitivity, random blood sugar, serum creatinine, blood urea nitrogen (BUN) and serum PSA were done at baseline. Patients with increased PSA above $4 \mathrm{ng} / \mathrm{mL}$ were given 4 weeks of antibiotic. If urine culture was positive, then sensitive antibiotic was given or otherwise empirically a fluroquinolone was given. After 6 weeks, PSA was repeated. Urine culture was repeated in those men whose urine culture showed bacterial growth initially. Patients were then evaluated for prostate volume, serum free PSA $\%$ and PSAD.

\section{mp-MRI}

All patients underwent mp-MRI using $1.5 \mathrm{~T}$ machine before TRUS-guided 12-core systematic biopsy (with additional biopsy core from suspected nodule on DRE). The PIRADS score version 2.0 was used to describe the mp-MRI findings. The PIRADS is mainly designed to improve detection, localization, characterization and risk stratification in patients with suspected CSPCa and its categories are based on the findings of mp-MRI (a combination of T2-weighted (T2W), diffusionweighted imaging (DWI) and dynamic contrast-enhanced (DCE) imaging). The PIRADS assesses the probability of finding CSPCa on a five-point Likert scale for each lesion [16-18]: PIRADS 1, very low (clinically significant cancer is highly unlikely to be present); PIRADS 2, low (clinically significant cancer is unlikely to be present); PIRADS 3, intermediate (the presence of clinically significant cancer is equivocal); PIRADS 4, high (clinically significant cancer is likely to be present); PIRADS 5, very high (clinically significant cancer is highly likely to be present). PIRADS scores 1 and 2 are considered as negative test and PIRADS scores 4 and 5 are considered as positive test. Using PIRADS 3 as negative test shall increase the specificity while using it as positive test shall increase the specificity. Additionally, it is possible to use PSA parameters to PIRADS 3 patients that can increase the accuracy of the test. These are free PSA and PSAD cut-off calculated from receiver operating characteristic (ROC) analysis of the study group. The sole objective behind combining PSA parameters cut-off value to PIRADS 3 category is to increase the specificity of the PIRADS test, so that we can altogether avoid TRUS-guided biopsy, if PIRADS test is negative.

\section{Prostate biopsy}

Histopathological results were reported as benign or malignant. If malignant type of malignancy, presence of high-grade prostate intraepithelial neoplasia (HGPIN), Gleason score, Gleason grade group, number of cores positive for cancer, length of core positive for cancer and percentage of core positive were taken into consideration.

If HGPIN was present, biopsy slides were being reviewed by a different pathologist. If on review, HGPIN was present on a single core, patient was advised to undergo repeat in following 3 years. Such cases were categorized as not having cancer in this study. However, if on review, HGPIN was present 
Table 1. Demographic Characteristics of Patients With Suspected PCa, Patients With PCa and Patients With BPH

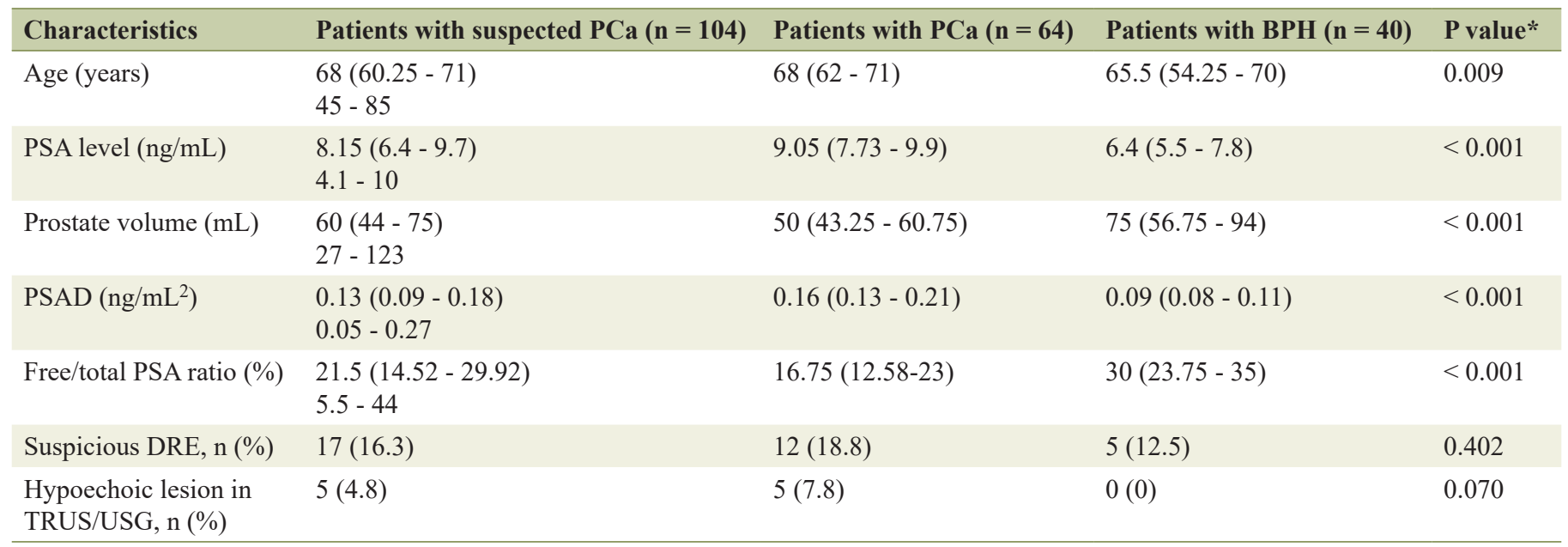

Data shown as median (interquartile range), range. Unless otherwise specified. *Comparison between patients with PCa and patients with BPH. $\mathrm{BPH}$ : benign prostatic hyperplasia; DRE: digital rectal examination; PCa: prostate cancer; PSA: prostate-specific antigen; PSAD: prostate-specific antigen density; TRUS: transrectal ultrasound; USG: ultrasound sonography.

on greater than or equal to two cores, then patient underwent repeat biopsy. If on repeat biopsy, only HGPIN was present, then it was categorized as not having cancer and patient was advised follow-up [6, 19].

Whenever there was a presence of atypical small acinar cell proliferation (ASAP) alone in biopsy, the slide was being reviewed by another pathologist. If ASAP was present, the patient underwent repeat biopsy.

\section{CSPC}

Presence of CSPCa in biopsy was defined by using "University College London definition 1": Gleason $\geq 4+3$ and/or cancer core length (CCL) $\max \geq 6 \mathrm{~mm}$ and/or total CCL $\geq 6 \mathrm{~mm}[20$, 21]. A five-point Likert reporting scale was used to designate prostate glands as highly unlikely (1), unlikely (2), equivocal (3), likely (4) and highly likely (5) to harbor CSPCa.

Based on whether there was presence or absence of cancer, the study population was divided into two groups: benign prostatic hyperplasia group (BPH) and PCa group. Based on whether there was presence or absence of CSPCa, the study population was divided into two groups: CSPCa group and non-CSPCa group (patients with clinically insignificant $\mathrm{PCa}$ or with $\mathrm{BPH}$ ). The latter categorization has been done as: 1) Both BPH and clinically insignificant PCa do not need definitive treatment rather than follow-up, whereas the CSPCa needs active management urgently; and 2) mp-MRI using PIRADS score only predicts the probability of having CSPCa from patients with $\mathrm{BPH}$ and clinically insignificant $\mathrm{PCa}$ combined.

\section{Statistical analysis}

Statistical analysis was done using SPSS Software (version 23). Pearson's Chi-square test was used to compare categorical variables and Mann-Whitney $U$ test for continuous variables. The best-fit ROC curve was calculated with area under the curve (AUC) estimates and 95\% confidence intervals (CIs). Univariate and multivariate analyses were performed using logistic regression analysis to determine significant predictors of PCa and CSPCa. Statistical significance was defined as $\mathrm{P}<$ 0.05 .

\section{Results}

A total of 104 patients with suspected PCa were divided into two groups: patients with $\mathrm{PCa}(\mathrm{n}=64)$ and patients with $\mathrm{BPH}$ $(\mathrm{n}=40)$. Table 1 shows comparison of clinical characteristics between patients with $\mathrm{PCa}$ and patients with $\mathrm{BPH}$. The median age $(\mathrm{P}=0.009)$, PSA $(\mathrm{P}<0.001)$, prostate volume $(\mathrm{P}<$ $0.001)$, PSAD $(\mathrm{P}<0.001)$ and free $\mathrm{PSA} \%(\mathrm{P}<0.001)$ were significantly different between the two groups. In patients with CSPCa, significantly higher median age $(\mathrm{P}=0.001)$, PSA level $(\mathrm{P}<0.001)$, PSAD $(\mathrm{P}<0.001)$ and significantly lower prostate volume $(\mathrm{P}<0.001)$ and free PSA\% were observed as compared to patients with non-CSPCa (Table 2).

Significantly higher proportion of patients with CSPCa showed PIRADS positive test compared to those with nonCSPCa $(86.4 \%$ vs. $53.3 \%, \mathrm{P}<0.001)$. Patients with individual PIRADS scores were also significantly different between the two groups (Table 3 ).

In ROC curve analysis, using a cut-off value of $0.12 \mathrm{ng} /$ $\mathrm{mL}^{2}$ for PSAD, the sensitivity and specificity of predicting CSPCa were 100\% (95\% CI: 91.96-100.00\%) and 80\% (95\% CI: $67.67-89.22 \%$ ), respectively. Using a cut-off value of $25 \%$ for free $\mathrm{PSA} \%$, the sensitivity and specificity of predicting CSPCa were 90.91\% (95\% CI: 78.33-97.47\%) and 58.33\% (95\% CI: 44.88-70.93\%), respectively (Table 4).

The univariate logistic regression analysis showed that factors including age, prostate volume, total PSA level, PSAD, 
Table 2. Comparison of Demographic Data Between Patients in CSPCa Group and Patients in Non-CSPCa Group (Having Insignificant Cancer or BPH)

\begin{tabular}{llll}
\hline Variables & CSPCa group $(\mathbf{n}=\mathbf{4 4})$ & Non-CSPCa group $(\mathbf{n}=\mathbf{6 0})$ & P value \\
\hline Age $($ years $)$ & $70(63.25-73.75)$ & $66(59-70)$ & 0.001 \\
PSA level $(\mathrm{ng} / \mathrm{mL})$ & $9.6(8.43-10)$ & $6.95(5.5-8.4)$ & $<0.001$ \\
Prostate volume $(\mathrm{mL})$ & $44.5(40.25-59.15)$ & $70(56-89.75)$ & $<0.001$ \\
PSAD $\left(\mathrm{ng} / \mathrm{mL}^{2}\right)$ & $0.20(0.16-0.22)$ & $0.10(0.08-0.12)$ & $<0.001$ \\
Free/total PSA ratio $(\%)$ & $14.25(11.08-18.73)$ & $28(21.25-33)$ & $<0.001$ \\
DRE positive, $\mathrm{n}(\%)$ & $7(15.9)$ & $10(16.7)$ & 0.918 \\
USG/TRUS positive, $\mathrm{n}(\%)$ & $4(9.1)$ & $1(1.7)$ & 0.080 \\
\hline
\end{tabular}

Data shown as median (interquartile range), range. Unless otherwise specified. CSPCa: clinically significant PCa; BPH: benign prostatic hyperplasia; DRE: digital rectal examination; PCa: prostatic cancer; PSA: prostate-specific antigen; PSAD: prostate-specific antigen density; TRUS: transrectal ultrasound; USG: ultrasound sonography.

Table 3. Comparison of PIRADS Score Between Patients in CSPCa Group and Patients in Non-CSPCa Group (Having Insignificant Cancer or BPH)

\begin{tabular}{llll}
\hline Multiparametric MRI results & CSPCa group $(\mathbf{n}=\mathbf{4 4 )}$ & Non-CSPCa group $(\mathbf{n}=\mathbf{6 0})$ & P value \\
\hline PIRADS $(+)^{*}$ & $38(86.4)$ & $32(53.3)$ & $<0.001$ \\
PIRADS score 1 & $2(4.5)$ & $7(11.7)$ & $<0.001$ \\
PIRADS score 2 & $3(6.8)$ & $21(35)$ & $22(36.7)$ \\
PIRADS score 3 & $6(13.6)$ & $7(11.7)$ \\
PIRADS score 4 & $11(25)$ & $3(5)$ & \\
PIRADS score 5 & $22(50)$ &
\end{tabular}

Data shown as $n(\%)$. *PIRADS score 3,4 or 5 is considered as a positive test. CSPCa: clinically significant prostate cancer; BPH: benign prostatic hyperplasia; PIRAD: Prostate Imaging Reporting and Data System; MRI: magnetic resonance imaging.

free PSA \% and PIRADS score were significant predictors of CSPCa. Multivariate logistic regression analysis showed that age, PSAD and free PSA\% were significant predictors of $\mathrm{PCa}$, while age and PSAD were significant predictors of CSPCa (Table 5).

In this study, four different criteria were used to define a positive or a negative test using PIRADS score and each criterion was compared for its diagnostic performance as a test to predict CSPCa (Table 6). The diagnostic performance of crite- ria 1, 2 and 3 for predicting CSPCa is shown in Table 7. When compared to criterion 1, criteria 2 and 3 showed comparable sensitivity $(88.64 \%, 85.19 \%$ and $82.81 \%$, respectively) and NPV $(84.85 \%, 84.31 \%$ and $73.81 \%$, respectively), and higher specificity $(46.67 \%, 86 \%$ and $77.50 \%$, respectively) and PPV $(54.93 \%, 86.79 \%$ and $85.48 \%$, respectively) in predicting CSPCa. The diagnostic performance of PIRADS score using criterion 1 showed higher sensitivity $(88.64 \%$ vs. $75 \%)$ and lower specificity ( $46.67 \%$ vs. $83.33 \%$ ) compared to criterion 4

Table 4. ROC Curve Analysis for Predicting Clinically Significant PCa for Each Factor

\begin{tabular}{llll}
\hline Parameters & AUC of ROC curve & $95 \%$ confidence interval & P value \\
\hline Age & 0.684 & $0.582-0.785$ & 0.001 \\
DRE & 0.496 & $0.383-0.609$ & 0.948 \\
USG/TRUS & 0.537 & $0.423-0.651$ & 0.519 \\
Serum PSA & 0.827 & $0.744-0.909$ & $<0.001$ \\
Prostate volume & 0.830 & $0.753-0.907$ & $<0.001$ \\
PSAD & 0.968 & $0.941-0.996$ & $<0.001$ \\
Free/total PSA ratio (\%) & 0.855 & $0.776-0.935$ & $<0.001$ \\
PIRADS score V2 & 0.822 & $0.734-0.909$ & $<0.001$ \\
\hline
\end{tabular}

AUC: area under the ROC curve; PCa: prostatic cancer; DRE: digital rectal examination; PIRADS: Prostate Imaging Reporting and Data System; PSA: prostate-specific antigen; PSAD: prostate-specific antigen density; ROC: receiver operating characteristic; TRUS: transrectal ultrasound; USG: ultrasound sonography. 
Table 5. Univariate and Multivariate Logistic Regression Analysis of Factors Associated With Clinically Significant PCa in Patients With Suspected PCa

\begin{tabular}{|c|c|c|}
\hline Parameters & Odds ratio $(95 \% \mathrm{CI})$ & P value \\
\hline \multicolumn{3}{|l|}{ Age } \\
\hline Univariate & $1.101(1.038-1.67)$ & 0.001 \\
\hline Multivariate & $1.157(1.008-1.327)$ & 0.038 \\
\hline $\operatorname{DRE}(+)$ & $0.946(0.329-2.717)$ & 0.918 \\
\hline TRUS/USG (+) & $0.169(0.018-1.573)$ & 0.118 \\
\hline Prostate volume & $0.916(0.882-0.950)$ & $<0.001$ \\
\hline \multicolumn{3}{|l|}{ Serum PSA } \\
\hline Univariate & $1.929(1.442-2.580)$ & $<0.001$ \\
\hline Multivariate & $0.930(0.571-1.515)$ & 0.772 \\
\hline \multicolumn{3}{|l|}{ PSAD } \\
\hline Univariate & $2.090(1.559-2.802)$ & $<0.001$ \\
\hline Multivariate & $2.033(1.466-2.819)$ & $<0.001$ \\
\hline \multicolumn{3}{|c|}{ Free/total PSA ratio (\%) } \\
\hline Univariate & $0.843(0.787-0.902)$ & $<0.001$ \\
\hline Multivariate & $0.939(0.846-1.043)$ & 0.243 \\
\hline PIRADS V2 & $5.542(2.04-15.053)$ & 0.001 \\
\hline
\end{tabular}

PCa: prostatic cancer; DRE: digital rectal examination; PIRADS: Prostate Imaging Reporting and Data System; PSA: prostate-specific antigen; PSAD: prostate-specific antigen density; TRUS: transrectal ultrasound; USG: ultrasound sonography.

in predicting CSPCa.

ROC curve analysis for predicting CSPCa using PIRADS score criteria 1, 2, 3 and 4 showed that the AUC values of criteria 2,3 and 4 were higher $(0.827,0.732$ and 0.792$)$ than criterion 1 (0.665) (Fig. 1 and Table 8).

\section{Discussion}

In the present study, significantly higher proportion of patients with CSPCa showed PIRADS positive test compared to those with non-CSPCa. Age, prostate volume, total PSA level, PSAD, free PSA\% and PIRADS score were significant pre- dictors of CSPCa. The AUC of ROC curve of PIRADS score for predicting CSPCa was 0.822 (95\% CI: 0.734 - 0.909; P < $0.001)$. This study used four criteria of PIRADS score to define positive test or negative test as shown in Table 6. Criteria 2, 3 and 4 demonstrated higher specificity and PPV in predicting CSPCa as compared to criterion 1 . The AUC values of criteria 2,3 and 4 were higher $(0.827,0.732$ and 0.792$)$ than criterion $1(0.665)$, suggesting them as better predictors of CSPCa. Therefore, for predicting CSPCa in a screening test, criterion 1 reported high sensitivity and low specificity, whereas use of criterion 4 decreased the number of false positive rates, thereby increasing the specificity and keeping sensitivity at same level. When compared with criterion 1, criteria 2, 3 and 4 demonstrated much higher diagnostic performance for predicting $\mathrm{CSPCa}$.

In this study, criterion 1 of PIRADS score for prediction of CSPCa showed sensitivity, specificity, PPV, NPV and accuracy of $88.64 \%, 46.67 \%, 54.93 \%, 84.85 \%$ and $64.42 \%$, respectively. Previous study reports are in concordance with these observations. Ahmed et al reported that sensitivity of PIRADS score for detecting CSPCa in patients with PSA up to $15 \mathrm{ng} / \mathrm{mL}$ was $93 \%$ with NPV of $89 \%$, specificity of $41 \%$ and with PPV of $51 \%$ [21]. Another study reported an AUC of 0.89 , and the NPV of PIRADS score of $\leq 2$ was $98 \%$. However, PPV of $49 \%$ raises a doubt about the PIRADS score being not able to predict the outcome. In addition, a few patients with a PIRADS score of $\leq$ 2 had CSPCa [22]. Thompson and colleagues (2016) found that when PIRADS score was used for predicting CSPCa, mp-MRI had sensitivity, specificity, PPV and NPV of $96 \%, 36 \%, 52 \%$ and $92 \%$, respectively [16]. A very recent study revealed that in patients with PSA between 4 and $10 \mathrm{ng} / \mathrm{mL}$, a PIRADS score $\geq$ 4 was the cut-off for predicting CSPCa $[18,20]$.

Many studies have shown the high NPV of mp-MRI for detecting both PCa and CSPCa using 12 cores, saturation or rectal prostectomy specimen as reference tests $[3,13-15,19$, 20]. The PPV which varies from $20 \%$ to $75 \%$ is strongly related to the reference test used, the patient population examined and the definition of a positive MRI. In this study, criterion 1 was defined PIRADS (version 2.0) score 3 as a positive, although PIRADS 3 is defined as intermediate, which means the presence of CSPCa is equivocal. Use of criterion 1 resulted in high sensitivity, low specificity, high NPV and low PPV for detection of CSPCa. Therefore, to evaluate mp-MRI as an early detection tool for CSPCa, the sensitivity (minimize missing

Table 6. PIRADS Score Criteria Used in the Study

\begin{tabular}{|c|c|c|c|}
\hline Criteria & Postive test & Negative test & Remarks \\
\hline 1 & Score 3,4 or 5 & Score 1 or 2 & Standard \\
\hline 2 & $\begin{array}{l}\text { Score } 4,5 \text { or }(3+ \\
\left.\text { PSAD }>0.12 \mathrm{ng} / \mathrm{mL}^{2}\right)\end{array}$ & $\begin{array}{l}\text { Score } 1,2 \text { or }(3+ \\
\left.\text { PSAD } \leq 0.12 \mathrm{ng} / \mathrm{mL}^{2}\right)\end{array}$ & Combining present study cut-off of PSAD with score 3 \\
\hline 3 & $\begin{array}{l}\text { Score } 4,5 \text { or }(3+ \\
\mathrm{fPSA} \leq 25.5 \%)\end{array}$ & $\begin{array}{l}\text { Score } 1,2 \text { or }(3+ \\
\text { fPSA }>25.5 \%)\end{array}$ & Combining present study cut-off of fPSA with score 3 \\
\hline 4 & Score 4 or 5 & Score 1,2 or 3 & $\begin{array}{l}\text { Score } 3 \text { taken as a negative test due to high rate of false positive in this study } \\
\text { when score } 3 \text { is taken as positive. }\end{array}$ \\
\hline
\end{tabular}

PIRADS: Prostate Imaging Reporting and Data System; fPSA: free/total PSA ratio; PSA: prostate-specific antigen; PSAD: prostate-specific antigen density. 
Table 7. Diagnostic Evaluation of PIRADS Score in Predicting CSPCa in Patients With Suspected PCa Using Criterion 1, Criterion 2 and Criterion 3

\begin{tabular}{|c|c|c|c|c|}
\hline \multirow{2}{*}{ Clinical group } & \multicolumn{4}{|c|}{ Clinically significant PCa with PIRADS scoring } \\
\hline & Criteria 1 & Criteria 2 & Criteria 3 & Criteria 4 \\
\hline Sensitivity & $88.64(75.44-96.21)$ & $85.19(72.88-93.38)$ & $82.81(71.32-91.10)$ & $75.00(59.66-86.81)$ \\
\hline Positive likelihood ratio & $1.66(1.28-2.15)$ & $6.08(3.03-12.20)$ & $3.68(2.05-6.61)$ & $4.50(2.49-8.13)$ \\
\hline Negative likelihood ratio & $0.24(0.10-0.58)$ & $0.17(0.09-0.33)$ & $0.22(0.13-0.39)$ & $0.30(0.18-0.51)$ \\
\hline Positive predictive value & $54.93(48.46-61.23)$ & $86.79(76.62-92.95)$ & $85.48(76.62-91.36)$ & $76.74(64.63-85.63)$ \\
\hline Negative predictive value & $84.85(70.15-93.03)$ & $84.31(73.74-91.14)$ & $73.81(61.61-83.19)$ & $81.97(72.91-88.48)$ \\
\hline Accuracy & $64.42(54.43-73.57)$ & $85.58(77.33-91.70)$ & $80.77(71.87-87.84)$ & $79.81(70.81-87.04)$ \\
\hline
\end{tabular}

Data shown as percentage $(95 \% \mathrm{CI})$. CI: confidence interval; fPSA: free/total prostate specific antigen ratio; PCa: prostate cancer; PIRADS: Prostate Imaging Reporting and Data System; PSAD: prostate-specific antigen density; CSPCa: clinically significant prostate cancer.

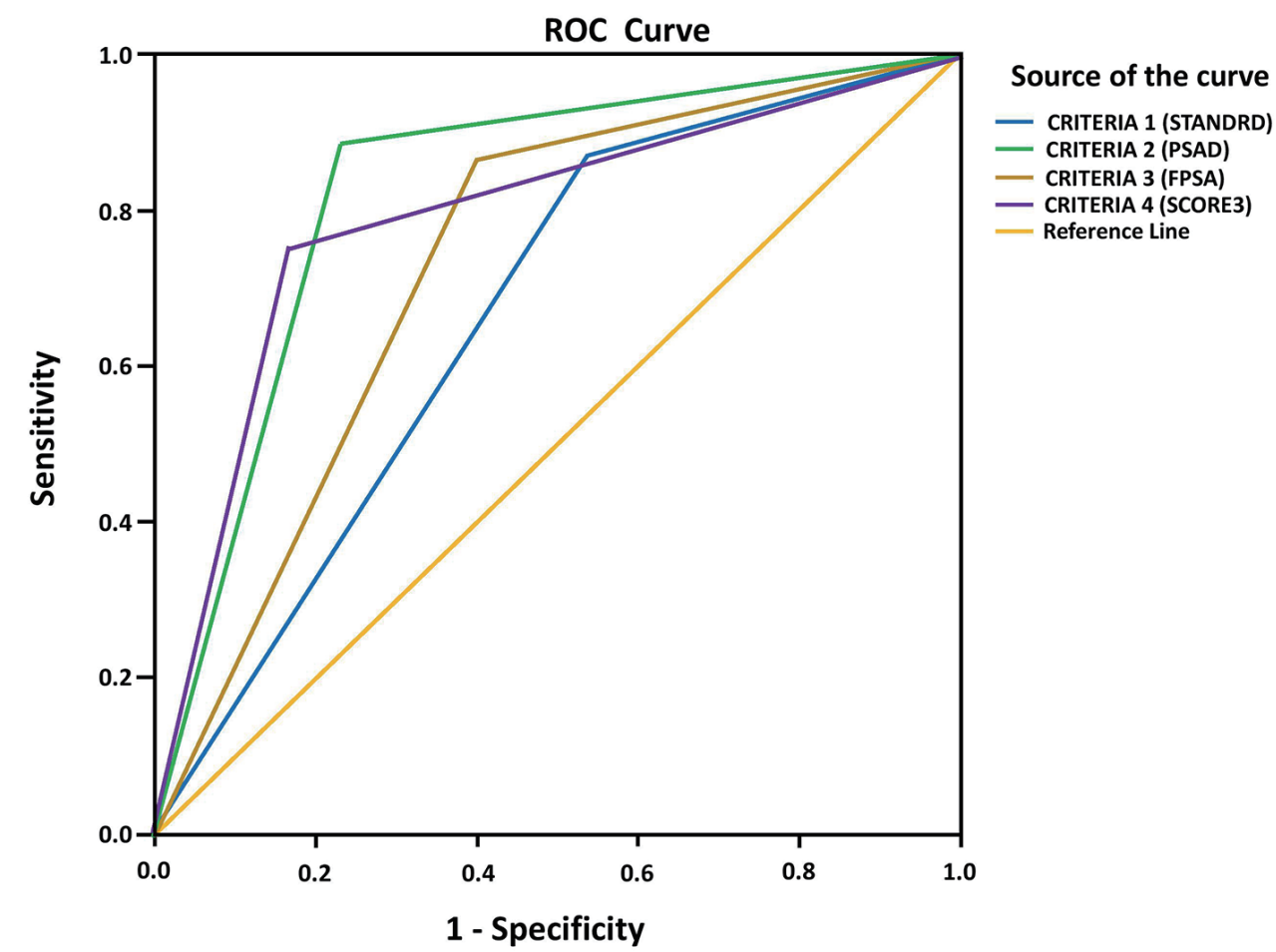

Figure 1. ROC curve analysis of PIRADS score criteria to determine CSPCa. ROC: receiver operating characteristic; PIRADS: Prostate Imaging Reporting and Data System; CSPCa: clinically significant prostate cancer.

Table 8. Summary of ROC Curve Analysis

\begin{tabular}{lllll}
\hline PIRADS score criteria & AUC of ROC curve & P value & \multicolumn{2}{c}{ 95\% confidence interval } \\
\cline { 4 - 5 } Criteria 1 & 0.665 & 0.004 & 0.561 & 0.769 \\
Criteria 2 & 0.827 & $<0.001$ & 0.743 & 0.910 \\
Criteria 3 & 0.732 & $<0.001$ & 0.634 & 0.829 \\
Criteria 4 & 0.792 & $<0.001$ & 0.699 & 0.884 \\
\hline
\end{tabular}

AUC: area under the ROC curve; PIRADS: Prostate Imaging Reporting and Data System; ROC: receiver operating characteristic. 
significant PCa) over specificity should be preferred and criterion 1 can be used.

When PIRADS score $\geq 4$ (criterion 4 ) was used as predictor of CSPCa instead of criterion 1, an increase of specificity, PPV and accuracy in predicting CSPCa was observed. This increase in diagnostic performance of criterion 4 is due to the absence of CSPCa in 22 out of 28 patients with PIRADS 3 score, which increased the number of false positives when criterion 1 was used. The comparison of AUC of ROC curve between criterion $1(0.665 ; \mathrm{P}=0.004)$ and criterion $4(0.792$; $\mathrm{P}<0.001$ ) shows better diagnostic performance of criterion 4 over criterion 1.

Engelhard et al study have evaluated the impact of PIRADS 3 score in differentiating equivocal lesions as malignant or benign and found that PIRADS 3 lesions revealed only benign conditions. PIRADS 3 score could not be confirmed as an absolute marker in patient clinical management care [18]. Another study which evaluated the significance of PIRADS score $\geq 4$ in identifying CSPCa showed that the sensitivity and specificity of PIRADS scoring were $77.0 \%$ and $73.8 \%$ for reader 1 and $77.3 \%$ and $71.4 \%$ for reader 2 , respectively [23]. These observations corroborate with results of previous studies which indicated increased specificity of PIRADS score to identify CSPCa when PIRADS $\geq 4$ (criterion 4 ) were used [16, 22]. The observations of this study signify the misleading nature of PIRADS 3 score and a substantial amount of false positive results. Therefore, to use PIRADS score as a screening test which needs more sensitivity and less specificity, criterion 1 can be used. However, to increase the diagnostic accuracy for identification of CSPCa and to avoid false positive results, criterion 4 should be used.

Using criterion 2 and criterion 3 for PIRADS score, this study demonstrated significantly increased sensitivity, specificity, PPV, NPV and overall accuracy of the test in predicting CSPCa. Such combinations of PIRADS score with PSA parameters are described sparsely in literature. Washino and colleagues assessed the value of the PIRADS version 2, for prostate $\mathrm{mp-MRI}$ and PSA parameters like PSA and PSAD in predicting biopsy outcome in biopsy naive patients who have suspected PCa. They found that when PIRADS score and PSAD were combined, a score of $\geq 4$ and PSAD $\geq 0.15 \mathrm{ng} / \mathrm{mL}^{2}$, or score of 3 and PSAD of $\geq 0.30 \mathrm{ng} / \mathrm{mL}^{2}$, was associated with the highest CSPCa detection rates (76-97\%) on the first biopsy. Men with PIRADS score of $\leq 3$ and PSAD of $<0.15 \mathrm{ng} / \mathrm{mL}^{2}$ may perform a role in avoiding unnecessary biopsies [24].

Vilanova and colleagues (2010) assessed the predictive value of mp-MRI and the free/total PSA ratio, alone and combined for PCa diagnosis. They found that the predictive model combining all variables was more accurate than each variable alone $\mathbf{9 5 . 2 \%}$ for combined vs. $73.5 \%$ for T2WI, $76.0 \%$ for MRS, $81.8 \%$ for DWI, $75.6 \%$ for DCE-MRI and $78.8 \%$ for free/total PSA ratio) [25].

Although in this study PIRADS, PSAD and free PSA\% were useful for predicting biopsy outcome separately, using PIRADS score 3 as positive test (criterion 1) has yielded a substantial number of false positive cases in predicting $\mathrm{CSPCa}$, thereby decreasing the accuracy of mp-MRI to $64.42 \%$. Use of criterion 3 (PIRADS score 3 with PSAD $>0.12 \mathrm{ng} / \mathrm{mL}^{2}$ as a positive test and PIRADS score 3 with $P S A D \leq 0.12 \mathrm{ng} / \mathrm{mL}^{2}$ as a negative test) has increased the accuracy of mp-MRI from $64.42 \%$ to $81.58 \%$ and specificity from $46.67 \%$ to $80 \%$, PPV from $54.93 \%$ to $75.51 \%$, and NPV from $84.85 \%$ to $87.27 \%$, respectively. Similarly, use of criterion 4 (PIRADS score 3 with free PSA $\% \leq 25.5 \%$ as a positive test and PIRADS score 3 with free PSA $\%>25.5 \%$ as a negative test) has increased the accuracy of mp-MRI from $64.42 \%$ to $76.92 \%$, specificity from $46.67 \%$ to $71.67 \%$, PPV from $54.93 \%$ to $75.51 \%$, and NPV from $84.85 \%$ to $86 \%$. The comparison of AUC of ROC curve among criterion $1(0.665 ; \mathrm{P}=0.004)$, criterion $2(0.827 ; \mathrm{P}<$ $0.001)$ and criterion $3(0.732 ; \mathrm{P}<0.001)$ shows statistically significant better diagnostic performance of criterion 2 and criterion 3 over criterion 1.

There are several limitations which need to be addressed. Firstly, this study considered only TRUS-guided systematic biopsy as the gold standard with which other parameters were compared. Secondly, although there are many definitions of CSPCa, authors considered the UCLA 1 definition [20, 21], considering other definitions may have affected the results.

\section{Conclusion}

Therefore, in patients above 40 years with LUTS and PSA in gray zone (4-10 ng/mL), combined mp-MRI (PIRADS score version 2.0), PSAD and free PSA\% can accurately predict presence or absence of CSPCa and can thereby exclude unnecessary biopsies.

\section{Acknowledgments}

None to declare.

\section{Financial Disclosure}

None to declare.

\section{Conflict of Interest}

None to declare.

\section{Informed Consent}

Written informed consent was obtained from each study patient before enrolment.

\section{Author Contributions}

All authors contributed to study design, recruitment of patients, data collection and manuscript preparation. SKB, ND and SJB additionally contributed to statistical analysis. All authors reviewed and approved the final version for submission. 


\section{References}

1. GLOBOCAN 2018 Prostate cancer fact sheet. Available from: https://gco.iarc.fr/today/data/factsheets/cancers/27Prostate-fact-sheet.pdf. Accessed on 30 July 2019.

2. Roobol MJ, Kranse R, Bangma $\mathrm{CH}$, van Leenders $\mathrm{AG}$, Blijenberg BG, van Schaik RH, Kirkels WJ, et al. Screening for prostate cancer: results of the Rotterdam section of the European randomized study of screening for prostate cancer. Eur Urol. 2013;64(4):530-539.

3. Pokorny MR, de Rooij M, Duncan E, Schroder FH, Parkinson R, Barentsz JO, Thompson LC. Prospective study of diagnostic accuracy comparing prostate cancer detection by transrectal ultrasound-guided biopsy versus magnetic resonance (MR) imaging with subsequent MRguided biopsy in men without previous prostate biopsies. Eur Urol. 2014;66(1):22-29.

4. Loeb S, Bjurlin MA, Nicholson J, Tammela TL, Penson DF, Carter HB, Carroll P, et al. Overdiagnosis and overtreatment of prostate cancer. Eur Urol. 2014;65(6):10461055.

5. Pinkhasov GI, Lin YK, Palmerola R, Smith P, Mahon F, Kaag MG, Dagen JE, et al. Complications following prostate needle biopsy requiring hospital admission or emergency department visits - experience from 1000 consecutive cases. BJU Int. 2012;110(3):369-374.

6. Wein AJ, Kavoussi LR, Partin AW, Craig A. Campbellwalsh urology. eleventh edition ed. Wein AJ, editor. Philadelphia: Elsevier; 2016.

7. Mazaheri Y, Shukla-Dave A, Muellner A, Hricak H. MR imaging of the prostate in clinical practice. MAGMA. 2008;21(6):379-392.

8. de Rooij M, Hamoen EH, Futterer JJ, Barentsz JO, Rovers MM. Accuracy of multiparametric MRI for prostate cancer detection: a meta-analysis. AJR Am J Roentgenol. 2014;202(2):343-351.

9. Purysko AS, Rosenkrantz AB, Barentsz JO, Weinreb JC, Macura KJ. PI-RADS Version 2: A pictorial update. Radiographics. 2016;36(5):1354-1372.

10. Barentsz JO, Richenberg J, Clements R, Choyke P, Verma S, Villeirs G, Rouviere O, et al. ESUR prostate MR guidelines 2012. Eur Radiol. 2012;22(4):746-757.

11. Barentsz JO, Weinreb JC, Verma S, Thoeny HC, Tempany CM, Shtern F, Padhani AR, et al. Synopsis of the PIRADS v2 guidelines for multiparametric prostate magnetic resonance imaging and recommendations for use. Eur Urol. 2016;69(1):41-49.

12. Steiger P, Thoeny HC. Prostate MRI based on PI-RADS version 2: how we review and report. Cancer Imaging. 2016;16:9

13. Wysock JS, Rosenkrantz AB, Huang WC, Stifelman MD, Lepor H, Deng FM, Melamed J, et al. A prospective, blinded comparison of magnetic resonance (MR) imaging-ultrasound fusion and visual estimation in the performance of MR-targeted prostate biopsy: the PROFUS trial. Eur Urol. 2014;66(2):343-351.

14. Hoeks CM, Schouten MG, Bomers JG, Hoogendoorn SP, Hulsbergen-van de Kaa CA, Hambrock T, Vergunst H, et al. Three-Tesla magnetic resonance-guided prostate biopsy in men with increased prostate-specific antigen and repeated, negative, random, systematic, transrectal ultrasound biopsies: detection of clinically significant prostate cancers. Eur Urol. 2012;62(5):902-909.

15. Chamie K, Sonn GA, Finley DS, Tan N, Margolis DJ, Raman SS, Natarajan S, et al. The role of magnetic resonance imaging in delineating clinically significant prostate cancer. Urology. 2014;83(2):369-375.

16. Thompson JE, van Leeuwen PJ, Moses D, Shnier R, Brenner P, Delprado W, Pulbrook M, et al. The diagnostic performance of multiparametric magnetic resonance imaging to detect significant prostate cancer. J Urol. 2016;195(5):1428-1435.

17. Hamoen EHJ, de Rooij M, Witjes JA, Barentsz JO, Rovers MM. Use of the Prostate Imaging Reporting and Data System (PI-RADS) for prostate cancer detection with multiparametric magnetic resonance imaging: a diagnostic meta-analysis. Eur Urol. 2015;67(6):1112-1121.

18. Engelhard K, Kuhn R, Osten A, Bogner K, Dworak A, Lubke L, Schneider F. Impact of magnetic resonance imaging-guided prostate biopsy in the supine position on the detection of significant prostate cancer in an inhomogeneous patient cohort. Scand J Urol. 2016;50(2):110-115.

19. Godoy G, Huang GJ, Patel T, Taneja SS. Long-term follow-up of men with isolated high-grade prostatic intraepithelial neoplasia followed by serial delayed interval biopsy. Urology. 2011;77(3):669-674.

20. Futterer JJ, Briganti A, De Visschere P, Emberton M, Giannarini G, Kirkham A, Taneja SS, et al. Can clinically significant prostate cancer be detected with multiparametric magnetic resonance imaging? A systematic review of the literature. Eur Urol. 2015;68(6):1045-1053.

21. Ahmed HU, El-Shater Bosaily A, Brown LC, Gabe R, Kaplan R, Parmar MK, Collaco-Moraes Y, et al. Diagnostic accuracy of multi-parametric MRI and TRUS biopsy in prostate cancer (PROMIS): a paired validating confirmatory study. Lancet. 2017;389(10071):815-822.

22. Grey AD, Chana MS, Popert R, Wolfe K, Liyanage SH, Acher PL. Diagnostic accuracy of magnetic resonance imaging (MRI) prostate imaging reporting and data system (PI-RADS) scoring in a transperineal prostate biopsy setting. BJU Int. 2015;115(5):728-735.

23. Park SY, Jung DC, Oh YT, Cho NH, Choi YD, Rha KH, Hong SJ, et al. Prostate cancer: PI-RADS version 2 helps preoperatively predict clinically significant cancers. Radiology. 2016;280(1):108-116.

24. Washino S, Okochi T, Saito K, Konishi T, Hirai M, Kobayashi Y, Miyagawa T. Combination of prostate imaging reporting and data system (PI-RADS) score and prostatespecific antigen (PSA) density predicts biopsy outcome in prostate biopsy naive patients. BJU Int. 2017;119(2):225233.

25. Vilanova JC, Barcelo-Vidal C, Comet J, Boada M, Barcelo J, Ferrer J, Albanell J. Usefulness of prebiopsy multifunctional and morphologic MRI combined with free-to-total prostate-specific antigen ratio in the detection of prostate cancer. AJR Am J Roentgenol. 2011;196(6):W715-722. 